\title{
The Log-logistic Regression Model with a Threshold Stress ${ }^{1}$
}

\author{
C.A.V. TOJEIRO ${ }^{2}$ F. LOUZADA-NETO ${ }^{3}$, Departamento de Estatística, UFSCar, \\ 13565-905 São Carlos, SP, Brasil.
}

\begin{abstract}
In this paper we propose an accelerated lifetime test model with threshold stress under a Log-logistic distribution to express the behavior of lifetimes and a general stress-response relationship. We present a sampling-based inference procedure of the model based on Markov Chain Monte Carlo techniques. We assume proper but vague priors for the parameters of interest. The methodology is illustrated on an artificial and real lifetime data set.
\end{abstract}

Keywords. ALT and Log-logistic models, Threshold Stress, Sampling-based inference, MCMC.

\section{Introduction}

Accelerated lifetime tests (ALT) are widely used as a means of getting information on the reliability of a manufactured product. They consist in submitting its units to higher stress levels than the usual working conditions, which provides useful and fast information about the lifetime distribution and other durability properties of the components (See, e.g., Mann et al. (1974), Abdel-Hameed et al (1984) and Nelson (1990)).

ALT models are composed of a probabilistic component, which is represented by a lifetime distribution such as exponential, Weibull, log-normal or log-logistic, among others, and by a stress-response relationship (SRR), which relates the mean lifetime with the stress levels. Common SRRs are the inverse power law, Eyring and Arrhenius models (Mann et al, 1974).

ALT users are particularly interested in determining the threshold stress (Hirose, 1993; Hagwood et al., 1999; Tojeiro et al., 2010), below which the lifetime of a product can be considered to be infinity, or a failure unlikely to occur. Knowing the threshold stress level, and not taking it into account may lead to serious errors in the estimates of other parameters of the model (Tojeiro et. al, 2004). We discuss in Section 4 a real ALT data set, given by Smith (1991), in which the threshold stress plays a crucial role. The exponential and weibull distributions are appropriate to fit data with a monotonic trend over time, with the hazard rate either increasing

\footnotetext{
${ }^{1}$ The authors are grateful to the editorial boarding and to the referees for their comments and suggestions, and to CAPES and to CNPq for their financial support.

${ }^{2}$ cynthia@ime.usp.br

${ }^{3}$ dfln@ufscar.br
}

Recebido em 26 Agosto 2010; Aceito em 28 Abril 2011. 
at higher values of $t$ or decreasing. However, these models are inadequate if the probability of an event at first increases, but after reaching a maximum value it tails off again, possibly to zero.

In order to make the model suitable for the case of non-monotonic hazard functions, in this paper we propose an ALT model with the log-logistic distribution to express the behavior of lifetimes. Besides sharing with the log-normal distribution a unimodal behavior of its hazard function, unlike the latter it has the advantage (like the Weibull and exponential models) of having simple algebraic expressions for the survivor and hazard functions.

The paper is organized as following. In the next Section we describe the model formulation. In Section 3 we discuss the hazard functions for the log-logistic distributions derived from the SRR and obtain the corresponding likelihood functions and we too develop a sampling-based analysis of the models via a Markov Chain Monte Carlo (MCMC) algorithm. The methodology is illustrated on an artificial data set and a real data set of ALT models in Section 4, where we also present the results of a simulation study that examines the coverage probabilities of the confidence intervals for the parameters based on the usual asymptotic theory. A brief discussion in Section 5 concludes the paper.

\section{Model Formulation}

Let $T$ be a random variable denoting the lifetimes with a probability distribution indexed by two parameters $\lambda$ and $\phi$, indicating the parameters of scale and shape, respectively. We define a general stress-response relationship (GSRR) relating the inverse of the scale parameter $1 / \lambda_{i}$ with a covariate vector of stress $\boldsymbol{z}$, which includes a unknown parameter vector of threshold stress $\boldsymbol{\omega}_{\mathbf{0}}$, such that, $\left(\boldsymbol{z}-\boldsymbol{\omega}_{\mathbf{0}}\right)$ is equal to $\mathbf{0}$, if $\boldsymbol{z}<\boldsymbol{\omega}_{\mathbf{0}}$ or equals to $\boldsymbol{z}-\boldsymbol{\omega}_{\mathbf{0}}$ if $\boldsymbol{z} \geq \boldsymbol{\omega}_{\mathbf{0}}$. With $m$ levels of the covariate $\boldsymbol{z}$, the GSRR is given by

$$
\lambda_{i}=\exp \left\{-\left(w_{i}+\boldsymbol{\alpha}^{T}+\boldsymbol{\beta}^{T}\left(\boldsymbol{z}-\boldsymbol{\omega}_{\mathbf{0}}\right)\right)\right\},
$$

where $i=1,2, \ldots, m$ and $-\infty<\boldsymbol{\alpha}, \boldsymbol{\beta}<\infty$ are vectors of unknown regression parameters. Although the GSRR (2.1) allows multiple covariates, which may occur in certain experiments, from now on we restrict ourselves for simplicity to the case of a single covariate $z$ and $\omega_{0}$, which is usual in survival analysis.

The GSRR given by (2.1) includes several SRR's used in engineering applications as particular cases:

i) when $\omega_{0}=0$ we have the general SRR defined in Achcar and Louzada-Neto (1992);

ii) the inverse power law model with a threshold stress is obtained for $w_{i}=0$, $\alpha=\log \left(\beta_{0}\right), \beta=-\beta_{1}$ and $\left(z-\omega_{0}\right)=\log \left(x_{i}-\omega_{0}\right)$, where $x_{i}, i=1 \ldots, m$, are the stress levels,

iii) Arrhenius model with a threshold stress corresponds to $w_{i}=0, \alpha=-\beta_{0}$, $\beta=\beta_{1},\left(z-\omega_{0}\right)=1 /\left(x_{i}-\omega_{0}\right)$; 
iv) if $w_{i}=-\log \left(x_{i}\right), \alpha=-\beta_{0}, \beta=\beta_{1}$ and $\left(z-\omega_{0}\right)=1 /\left(x_{i}-\omega_{0}\right)$, we have Eyring's model with a threshold stress.

If, in addition, $\omega_{0}=0$ in (ii), (iii) and (iv), we get the usual inverse power law, Arrhenius and Eyring models, respectively.

Although, in principle, the methodology developed in this paper can be applied to any SRR included in (2.1), we restrict ourselves to the case of a inverse power law model with a threshold stress as in (ii), that is,

$$
\lambda_{i}=\exp \left\{-\log \left(\beta_{0}\right)+\beta_{1} \log \left(x_{i}-\omega_{0}\right)\right\} .
$$

\section{Inference}

For inference we adopt a fully Bayesian approach. The likelihood function, prior distributions for the parameters in the model and details of the MCMC algorithm are described below.

\subsection{Likelihood function}

Considering a scenario characterized by submitting $m$ groups of $n_{i}$ items, $i=$ $1, \ldots, m$, under $m$ constant and fixed stress covariates $z_{i}$ (hereafter referred to as stress levels) associated with the failure times $t_{i j}, j=1, \ldots, n_{i}$, the maximum likelihood estimates (MLEs) of the parameters can be obtained by direct maximization of the log-likelihood function

$$
\log L=\sum_{i=1}^{m} \sum_{j=1}^{n_{i}} \delta_{i j} \log h\left(t_{i j} \mid z_{i}\right)-H\left(t_{i j} \mid z_{i}\right)
$$

where $\delta_{i j}=1$ if $t_{i j}$ is an observed failure time and $\delta_{i j}=0$ if $t_{i j}$ is a right-censored observation, and $H(t \mid z)=\int_{0}^{t} h(u \mid z) d u$ is the cumulative baseline hazard function (Lawless, 2002). Right-censoring is the only form of censoring considered here, though the principles of the proposed maximum likelihood analysis are certainly not dependent on such restriction.

Let $T$ be a lifetime random variable with a log-logistic distribution with parameters $\lambda>0$ and $\phi>0$, respectively (Lawless, 2002). The corresponding hazard function using the inverse power law model with threshold stress for a covariate $z$, as described in ii) of Section 2 is given by

$$
h(t \mid z)=\frac{\phi \lambda^{\phi} t^{\phi-1}}{1+(\lambda t)^{\phi}}
$$

where $\lambda_{i}=1 / \mu_{i}=\exp \left\{-\log \left(\beta_{0}\right)+\beta_{1} \log \left(x_{i}-\omega_{0}\right)\right\}$.

Given the data from $m$ random stress levels with a censoring scheme as described above, it follows from (3.1) and (3.2) that the kernel of the likelihood function for 
the parameters $\beta_{0}, \beta_{1}, \omega_{0}$ and $\phi$, under the $\mathrm{i}$-th stress level $x_{i}$, is given by

$$
L\left(\beta_{0}, \beta_{1}, \omega_{0}, \phi\right) \alpha \prod_{i=1}^{m} \prod_{j=1}^{n_{i}}\left[\frac{\phi \lambda_{i}^{\phi} t_{i j}^{\phi-1}}{1+\left(\lambda_{i} t_{i j}\right)^{\phi}}\right]^{\delta_{i j}}\left(\frac{1}{1+\left(\lambda_{i} t_{i j}\right)^{\phi}}\right) .
$$

\subsection{Sampling-based inference}

Inferences about the parameters can be based on a fully Bayesian approach, where their marginal posterior densities, which can be obtained by integrating the joint posterior density. In our case, however, it is not possible to obtain analytical solutions for the integrals. In order to overcome this problem we use the Gibbs sampler approach, which is an iterative procedure of a broad class of methods generically named Markov Chain Monte Carlo (MCMC). Interested readers can refer to Gelfand and Smith (1990) and Gilks et al. (1996), which describe many practical aspects of MCMC. This method is applicable in situations where one is not able to generate samples directly from the joint posterior density. However, the full conditional posterior densities for the parameters are required in each step of the iterative sampling-based algorithms.

In order to avoid numeric instability, we follow Davison and Hinkey (1997) and consider the parametrization $\beta_{0}, \beta_{1}, \omega_{0}=x_{1}-\exp (\rho)$ and $\phi$,for this model. We first specify a prior density for $\beta_{0}, \beta_{1}, \rho$ and $\phi$. For simplicity, we assign independent normal prior distributions $N\left(\mu_{i}, \sigma_{i}^{2}\right)$ with $\mu_{i}=0$ and large $\sigma_{i}^{2}, i=1, \ldots, 3$ for the parameters $\beta_{0}, \beta_{1}$ and $\rho$. Since the parameter $\phi$ is non-negative, a prior distribution with support over the positive line is desired. We consider, for instance, a gamma distribution with parameters $(q, v)$ for $\phi$ in the Log-logistic case. The hyperparameter values have been chosen in order to represent locally non informative priors. In order to give support to our choice of the distributions, a sensitivity analysis of the hyperparameters has been carried out, which indicated very close results for the parameter estimations under different choices of the hyperparameters.

Then, combining the prior densities for $\beta_{0}, \beta_{1}, \rho$ and $\phi$ with (3.3), we obtain that their joint posterior density is given by

$$
\begin{aligned}
\pi\left(\beta_{0}, \beta_{1}, \rho, \phi, \mid \text { data }\right) \alpha \prod_{i=1}^{m} \prod_{j=1}^{n_{i}} & {\left[\frac{\phi \lambda_{i}^{\phi} t_{i j}^{\phi-1}}{1+\left(\lambda_{i} t_{i j}\right)^{\phi}}\right]^{\delta_{i j}}\left(\frac{1}{1+\left(\lambda_{i} t_{i j}\right)^{\phi}}\right) } \\
& \exp \left(-\frac{\beta_{0}^{2}}{2 \sigma_{1}^{2}}-\frac{\beta_{1}^{2}}{2 \sigma_{2}^{2}}-\frac{\rho^{2}}{2 \sigma_{3}^{2}}-v \phi\right) \phi^{q-1},
\end{aligned}
$$

which can also be written as

$$
\begin{aligned}
\pi\left(\beta_{0}, \beta_{1}, \rho, \phi, \mid \text { data }\right) \alpha \exp & \left\{\sum_{i=1}^{m} \sum_{j=1}^{n_{i}} \delta_{i j} \log \left[\frac{\phi \lambda_{i}^{\phi} t_{i j}^{\phi-1}}{1+\left(\lambda_{i} t_{i j}\right)^{\phi}}\right]-\log \left(1+\left(\lambda_{i} t_{i j}\right)^{\phi}\right)\right. \\
& \left.-\left(\frac{\beta_{0}^{2}}{2 \sigma_{1}^{2}}+\frac{\beta_{1}^{2}}{2 \sigma_{2}^{2}}+\frac{\rho^{2}}{2 \sigma_{3}^{2}}+v \phi\right)+(q-1) \log \phi\right\} .
\end{aligned}
$$

Since the full conditional densities for $\beta_{0}, \beta_{1}, \rho$, and $\phi$ (described in Appendix A) do not belong to any known parametric family, in order to generate samples 
we need to implement an acceptation/rejection algorithm. In our case we use the adaptative rejection metropolis sampling algorithm (ARMS) introduced by Gilks et al.(1995). The ARMS algorithm is a generalization of the method of adaptative rejection sampling algorithm (ARS) (Gilks and Wild, 1992), which includes a metropolis step to accommodate non-log-concavity in the density to be sampled. The adaptative rejection metropolis sampling algorithm is implemented in the software BUGS (Spiegehalter et al., 1997).

\section{Data Analysis}

In this section the proposed methodology is applied to two data set. The first one is an artificial data set while the second one is a real numerical example extracted from Smith (1991). We also present the results of a simulation study performed in order to study the coverage probabilities of the confidence intervals for the model parameters in presence of small or moderated sized samples if the usual frequentist theory were considered.

\subsection{Artificial data}

The artificial data set was based on a sample generated from the log-logistic distribution using the inverse power law model (3.2). The parameters have been given fixed values $\beta_{0}=2.5, \beta_{1}=2.0, \phi=3$ and $\omega_{0}=4.8$. Three stress levels have been fixed at $x=5,10,15$ with 100 non-censored observations at each level. By means of graphical techniques we can roughly determine the shape of the hazard function and find out whether the shape parameter depends on covariates or if it remains unchanged for all stress levels (Lawless, 2002). The shape is the same for all stress levels if one expects the plots of the estimated survival functions to be parallel straight lines (Nelson, 1990). Since this is roughly the case in our situation, as shown in Figure 1, a good fit by a log-logistic model with a constant shape parameter is suggested (Lawless, 2002).

We have used the software CODA (Best et al., 1995) for monitoring the behavior of the chains. The Raftery-Lewis diagnostic suggested 8.000 iterations to reach the convergence for the parameters. Running BUGS again with these values has detected convergence by all implemented criteria in CODA. To study the sensitivity in the parameters estimates, we have considered some different hyperparameter values in the suggested priors. These were $N\left(0, \sigma_{i}^{2}\right)$ with $\sigma_{i}^{2}=10^{2}, 10^{3}, 10^{4}$ for $i=1, \ldots, 3$ and $\Gamma(q, v)$ with $q=10^{-1}$ and $v=10^{-2}, 10^{-3}$. Since similar estimates have been obtained for all cases, we only show the results for $\sigma_{i}^{2}=10^{4}$ and $b=10^{-3}$.

The parameters estimates of the model based on our sample-based analysis and their 95\% HDP intervals by considering the Gibbs sampler algorithm are given by: $\hat{\beta}_{0}=2.59(2.26 ; 3.11), \hat{\beta}_{1}=2.16(1.98 ; 2.58), \hat{\omega}_{0}=4.63(4.48 ; 4.74)$ and $\hat{\phi}=3.32$ $(2.98 ; 3.89)$, respectively. 

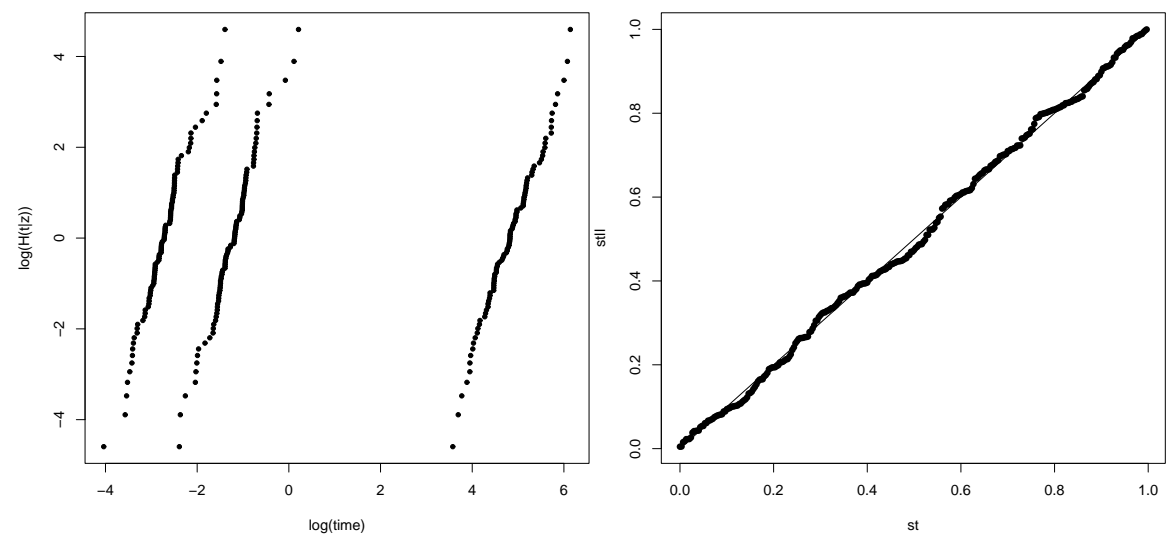

Figure 1: Left panel: linearized $H(t)$ for artificial data. Right panel: KaplanMeier(st) against log-logistic model(stll) for artificial data.

\subsection{Kvelar fibres data}

As a real numerical example we have considered the data extracted Table 2 of Smith (1991), which consists of failure times of Kvelar 49 fibres loaded at four different stress levels. For this kind of data the log-logistic distribution is widely used. We have considered the test performed at $m=3$ levels of the stress; $x_{i}=23.4,25.5$ and 27.6 MPa for $i=1, \ldots, 3$, with $n_{i}=21,25$ and 24 observations at each level. In the level 23.4 we have 11 censured observations. A good fit by a log-logistic model is suggested maybe of the plot of $S(t)$-empirical Kaplan-Meier Survival function against log-logistic model(Figure 2-a)for all levels and 2-b)for each level of stress. We can also observe a good fit through of the plot of Normalized randomized quantile residuals against $N(0,1)$ quantiles (Figure 2-c). Also the plot of $\log \hat{H}(t \mid z)$ against $\log t$, given in Figure 2-d, turned out to be roughly linear and parallel, where $\hat{H}(t \mid z)$ is the empirical cumulative hazard function (Lawless, 2002), a constant shape parameter $\phi$ for the failure time was suggested.

The parameters estimates of the model based on our sample-based analysis and their 95\% HDP intervals by considering the Gibbs sampler algorithm are given by: $\hat{\beta}_{0}=18.74(15.76 ; 23.86), \hat{\beta}_{1}=5.77(3.82 ; 7.40), \hat{\omega}_{0}=21.66(18.09 ; 24.13)$ and $\hat{\phi}=1.306(1.034 ; 1.606)$, respectivelly.

Again we have used the software CODA (Best et al. 1995) for monitoring the behavior of the chains. The Raftery-Lewis diagnostic suggested 12,000 iterations to reach the convergence for the parameters. Running BUGS again with these values has detected convergence by all implemented criteria in CODA. To study the sensitivity in the parameters estimates, we have considered some different hyperparameter values in the suggested priors. These were $N\left(0, \sigma_{i}^{2}\right)$ with $\sigma_{i}^{2}=10^{2}, 10^{3}, 10^{4}$ for $i=1, \ldots, 3$ and $\operatorname{Gamma}(a, b)$ with $a=10^{-1}$ and $b=10^{-2}, 10^{-3}$. Since similar estimates have been obtained for all cases, we only show the results for $\sigma_{i}^{2}=10^{4}$ 
and $b=10^{-3}$.
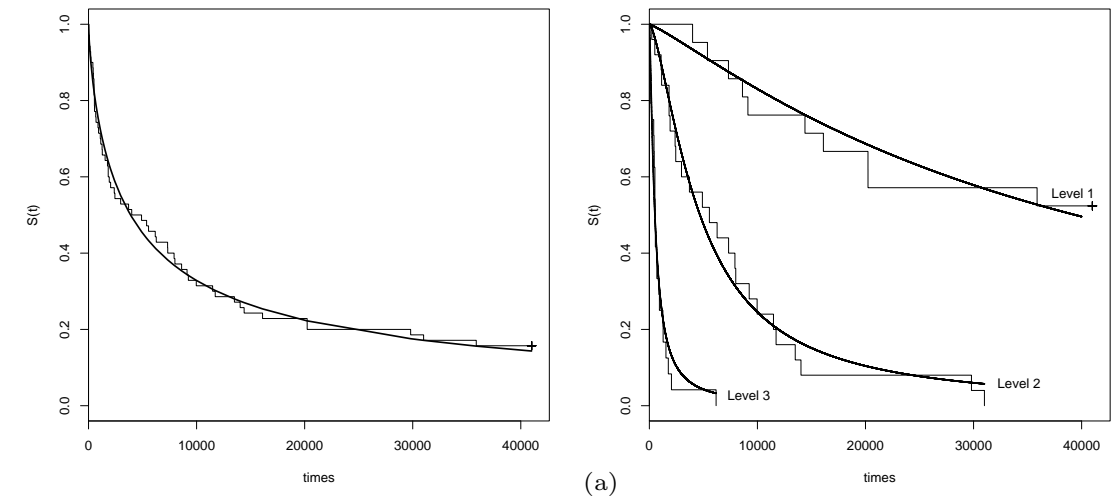

(b)
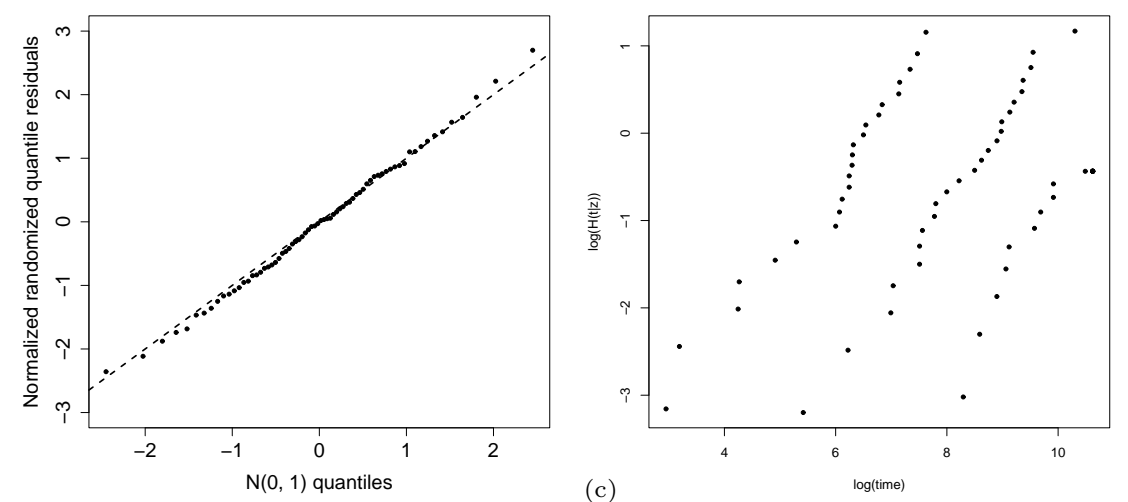

(d)

Figure 2: (a): Empirical survival function with the log-logistic distribution for all levels and (b): for each levels (Kvelar). (c): Quantiles (Kvelar). (d): Linearized $\mathrm{H}(\mathrm{t})$ (Kvelar)

\subsection{Coverage probabilities}

In this section we realized a simulation studies, where we studed the coverage probabilities of the usual aymptotic confidence intervals, with the objective of analizing the frequentist theory for small or moderate data sets. To examine the frequentist properties we constructed the confidences intervals for all the parameters and calculated they coverage probabilities.

From the frequentist point of view, inference for the parameter vector $\psi=$ $\left(\beta_{0}, \beta_{1}, \omega_{0}, \phi\right)$ can be based on large sample properties of the MLEs, which leads to 
(Sen and Singer, 1993)

$$
\frac{(\widehat{\boldsymbol{\psi}}-\boldsymbol{\psi})}{I^{-1 / 2}(\widehat{\boldsymbol{\psi}})} \stackrel{D}{\longrightarrow} N\left(0, I_{4}\right)
$$

where $I^{-1 / 2}(\widehat{\boldsymbol{\psi}})$ denotes the observed information matrix of $\boldsymbol{\psi}$ evaluated at the MLEs and $I_{4}$ is the identity matrix of dimension four. Although this is an standard asymptotical method, we shall see bellow that it does not lead to very accurate results for small or moderate samples sizes, which is usually the case in reliability studies. We check the behavior of the asymptotic theory for small or moderate data sets, by performing a small-scale simulation study for examining the coverage probabilities of the confidence intervals for the parameters obtained via (3.3).

Samples have been generated according to a study performed at three covariate levels, $x=5,10,15$ with $n=10,30,100$ non-censored items at each level, and the log-logistic distribution (3.2) has been assumed for the lifetimes with scale and shape parameters $\lambda_{i}=1 / \mu_{i}=\exp \left\{-\log \left(\beta_{0}\right)+\beta_{1} \log \left(x_{i}-\omega_{0}\right)\right\}$, and $\phi$, respectively. The parameter values have been fixed at $\beta_{0}=2.5, \beta_{1}=2, \omega_{0}=4.8$, and $\phi=3$. A thousand samples have been generated for each case. Table 1 shows the variation in coverage of nominal $95 \%$ confidence intervals according to the sample size. The $95 \%$ confidence interval for the nominal coverage probability of 0.95 based on a sample of size equals to a thousand observations is given by $(0.929,0.961)$. If a confidence interval has exact coverage of 0.95 , roughly $95 \%$ of the observed coverages should be inside these bounds. There is clear under-coverage of the confidence intervals for small and moderate sized samples. Such findings give evidence for the need of more adequate procedures for small or moderate sized samples, such as the samplingbased procedure developed here.

Table 1: Coverage probabilities of nominal $95 \%$ confidence intervals.

\begin{tabular}{ccccc}
\hline \hline$n$ & $\beta_{0}$ & $\beta_{1}$ & $\omega_{0}$ & $\phi$ \\
\hline 10 & 0.88 & 0.89 & 0.86 & 0.83 \\
\hline 30 & 0.91 & 0.90 & 0.91 & 0.87 \\
\hline 100 & 0.95 & 0.96 & 0.94 & 0.91 \\
\hline \hline
\end{tabular}

\section{Final Comments}

The model considered in this paper can be extended in several directions. In principle, a general model, knowing with a hybrid hazard regression model with threshold stress which includes the proportional hazards and the accelerated failure time models as particular cases, can be considered to express the behavior of lifetimes. Our model is defined as

$$
h(t \mid \boldsymbol{z})=g_{1}\left(\boldsymbol{\alpha}_{\mathbf{0}}^{T}\left(\boldsymbol{z}-\boldsymbol{\omega}_{\mathbf{0}}\right)\right) h_{0}\left(g_{2}\left(\boldsymbol{\alpha}_{\mathbf{1}}^{T}\left(\boldsymbol{z}-\boldsymbol{\omega}_{\mathbf{0}}\right)\right) t ; \boldsymbol{\phi}\right),
$$


where $g_{1}(),. g_{2}($.$) are known positive monotone functions that assume the value 1$ when their arguments are zero, $\boldsymbol{z}$ is a covariate vector, $\boldsymbol{\alpha}_{\mathbf{0}}, \boldsymbol{\alpha}_{\mathbf{1}}$ and $\boldsymbol{\phi}$ are vectors of unknown regression parameters, $\boldsymbol{\omega}_{\mathbf{0}}$ is the unknown parameter vector of threshold stress, as described in Section 2, and $h_{0}($.$) denotes the baseline hazard function.$ From the parametric point of view, if we assume $g_{1}(\cdot)=g_{2}(\cdot)=g(\cdot)$ and

$$
h_{0}(u ; \phi)=\frac{1}{1+u^{\phi}},
$$

with $u=\mu t$, model (2.1) corresponds to the hazard function of a random variable with a log-logistic distribution with the scale parameter depending on covariates (Kalbfleisch and Prentice, 1980).

Other distributions too can be used besides the log-logistic. One can also deal with situations that involve more than one covariate, some of which may admit thresholds. Another possibility is to consider different censoring schemes other than type right-censoring. The latter was used in this paper because it is the one that typically appears in industrial experiments. Other censoring schemes like random censoring may be used in applications such as survival experiments. We hope to report on results related to these extensions in future work.

We have developed a Bayesian approach for the analysis of the models. Although, we have adopted Normal priors throughout, the methodology employed is quite general, and the prior specifications could be adopted relatively easily. Inference for the model parameters is based on MCMC Methods which worked well in application.

\section{Appendix}

The full conditional posterior densities for $\beta_{0}, \beta_{1}, \rho$ and $\phi$ are given by

$$
\begin{gathered}
\pi\left(\beta_{0} \mid \beta_{1}, \rho, \phi\right) \alpha \exp \left[\sum_{i=1}^{m} \sum_{j=1}^{n_{i}} \delta_{i j}\left(-\phi \beta_{0}\right)-\left(\delta_{i j}+1\right) \log \left(1+\left(\lambda_{i} t_{i j}\right)^{\phi}\right)-\frac{\beta_{0}^{2}}{2 \sigma_{1}^{2}}\right], \\
\pi\left(\beta_{1} \mid \beta_{0}, \rho, \phi\right) \alpha \exp \left[\sum_{i=1}^{m} \sum_{j=1}^{n_{i}} \delta_{i j}\left[\phi \beta_{1} \log \left(x_{i}-x_{1}+\exp (\rho)\right)\right]\right. \\
\left.-\left(\delta_{i j}+1\right) \log \left(1+\left(\lambda_{i} t_{i j}\right)^{\phi}\right)-\frac{\beta_{\prime 1}^{2}}{2 \sigma_{2}^{2}}\right] \\
\pi\left(\rho \mid \beta_{0}, \beta_{1}, \phi\right) \alpha \exp \left[\sum_{i=1}^{m} \sum_{j=1}^{n_{i}} \delta_{i j}\left[\phi \beta_{1} \log \left(x_{i}-x_{1}+\exp (\rho)\right)\right]\right. \\
\left.-\left(\delta_{i j}+1\right) \log \left(1+\left(\lambda_{i} t_{i j}\right)^{\phi}\right)-\frac{\rho}{2 \sigma_{3}^{2}}\right]
\end{gathered}
$$


and

$$
\begin{array}{r}
\pi\left(\phi \mid \beta_{0}, \beta_{1}, \rho\right) \alpha \exp \left[\sum_{i=1}^{m} \sum_{j=1}^{n_{i}} \delta_{i j} \log \left[\phi \lambda_{i}^{\phi} t_{i j}^{\phi-1}\right]-\left(\delta_{i j}+1\right) \log \left(1+\left(\lambda_{i} t_{i j}\right)^{\phi}\right)\right. \\
-v \phi+(q-1) \log \phi] .
\end{array}
$$

\section{References}

[1] M. Abdel-Hameed, E. Cinlar, J. Quinn, "Reliability Theory and Models: Stochastic Failure Models, Optimal Maintenance Policies, Life Testing and Structures", Academic Press, New York, 1984.

[2] J. Achcar, F. Louzada-Neto, A Bayesian approach for accelerated life tests considering the Weibull distribution, Computational Statistics, 7, (1992) 355368.

[3] N.G. Best, M. K. Cowles, S.K. Vines, "CODA: Convergence Diagnostic and Output Analysis Software for Gibbs Sampling Output, Version 0.3. MRC" Biostatistics Unit, Cambridge, 1995.

[4] A.C. Davison, D.V. Hinkley, "Bootstrap Methods and their Applications", Cambridge University Press, Cambridge, 1997.

[5] A.E. Gelfand, A.F.M. Smith, Sampling-based approaches to calculating marginal densities. Journal of the Americam Statistical Society, B, 85, (1990) 398-409.

[6] W.R. Gilks, P. Wild, Adaptative rejection sampling for Gibbs sampling. Applied Statistics, 41, (1992) 337-348.

[7] W.R. Gilks, N.G. Best, K.K.G. Tan, Adaptative rejection metropolis sampling with Gibbs sampling, Applied Statistics, 44, (1995) 455-472.

[8] W.R. Gilks, S. Richardson, D.J. Spiegelhalter, "Markov Chain Monte Carlo in Practice", Chapman \& Hall, London, 1996.

[9] H. Hirose, Estimation of threshold stress in accelerated life-testing. IEEE Transactions on Reliability, 42, (1993) 650-657.

[10] C. Hagwood, R. Clough, R. Fields, Estimation of the threshold-stress for the Weibull inverse power law, IEEE Transactions on Reliability, 48, (1999) 176181.

[11] J.D. Kalbfleisch, R.L. Prentice, "The Statistical Analysis of Failure Time Data", Jonh Wiley and Sons, New York, 1980.

[12] J.F. Lawless, "Statistical Models and Methods for Lifetime Data", Jonh Wiley \& Sons, New York, 2002. 
[13] F. Louzada-Neto, C.A.V. Tojeiro, G.C. Perdoná, A hybrid Hazard model with threshold stress: the Weibull case, Advances and Applications in Statistical Sciences, 1, (2010) 145-155.

[14] N.R. Mann, R.E. Schafer, N.D. Singpurwalla, "Methods for Statistical Analysis of Reliability and Life Data", Jonh Wiley and Sons, New York, 1974.

[15] W. Nelson, "Accelerated Testing: Statistical Models, Test Plans and Data Analysis". Jonh Wiley and Sons, New York, 1990.

[16] P.K. Sen, J.M. Singer, "Large Sample Methods in Statistics: an Introduction with Applications", Chapman \& Hall, New York, 1993.

[17] R.L. Smith, Weibull regression models for reliability data, Reliability Engineering and System Safety, 34, (1991) 55-77.

[18] D. Spiegehalter, A. Thomas, N. Best, et al. "BUGS (Bayesian Inference Using Gibbs Sampling Version 0.5)", Cambridge, England, 1997.

[19] R.L. Prentice, Linear rank tests with right censored data, Biometrika, 65, (1978) 167-179.

[20] C.A.V. Tojeiro, F. Louzada-Neto, H. Bolfarine, A Bayesian analysis for accelerated lifetime tests under an exponential power law model with threshold stress, Journal of Applied Statistics, 31, (2004) 685-691. 